\title{
Convective heat transfer analysis on Prandtl fluid model with peristalsis
}

\author{
A. Alsaedi ${ }^{\mathrm{a}}$, Naheed Batool ${ }^{\mathrm{b}}, \mathrm{H}$. Yasmin ${ }^{\mathrm{b}, *}$ and T. Hayat ${ }^{\mathrm{a}, \mathrm{b}}$ \\ ${ }^{a}$ Department of Mathematics, Faculty of Science, King Abdulaziz University, Jeddah, Saudi Arabia \\ ${ }^{\mathrm{b}}$ Department of Mathematics, Quaid-I-Azam University, Islamabad, Pakistan
}

\begin{abstract}
The effects of magnetohydrodynamic (MHD) on peristaltic transport of Prandtl fluid in a symmetric channel have been studied under the assumptions of long wave length and low-Reynolds number. Channel walls are considered compliant in nature. Series solutions of axial velocity, stream function and temperature are given by using regular perturbation technique for small values of Prandtl fluid parameter. The effects of physical parameters on the velocity, streamlines and temperature are examined by plotting graphs.
\end{abstract}

Keywords: Prandtl fluid, magnetic field, compliant walls, convective conditions

\section{Introduction}

Considerable attention has been focused on the peristaltic transport of Newtonian and non-Newtonian fluids through tubes/channels in past few decades. This process is quite important for fluid transport in view of theoretical and industrial perspectives. Peristaltic transport has great importance in many biological systems such as food swallowing through the esophagus, circulation of blood in small blood vessels and the flows of many other glandular ducts etc. In addition peristaltic pumping has many industrial applications involving biomechanical systems such as heart-lung machine, finger and roller pumps etc. Some significant literature illustrating Newtonian and non-Newtonian fluid with/without peristaltic transport are given in Refs. [1-6]. Recently it is a well-accepted fact that the peristaltic flows of magnetohydrodynamic (MHD) fluids are important in medical sciences and bioengineering. The MHD characteristics are useful in the development of magnetic devices, hyperthermia and

*Corresponding author: H. Yasmin, Department of Mathematics, Quaid-I-Azam University 45320, Islamabad 44000, Pakistan. Tel.: +92 51 90642172; E-mail: qau2011@ gmail.com. blood reduction during surgeries and cancer tumor treatment. Also the effect of magnetic field on viscous fluid has been reported for treatment of the pathologies e.g. Gastroenric pathologies, rheumatisms, constipation and hypertension that can be treated by placing one electrode either on the back or on the stomach and the other on the sole of the foot; this location will induce a better blood circulation. Hence several researchers have discussed the peristalsis with magnetic field effects [7-11].

The idea of complaint nature of channel walls in peristalsis was initiated by Kramer [12], where he covered an underwater object with rubber and found considerable reductions in the drag. Experiments have been conducted to study other flows past compliant boundaries, blood flow in arteries, dolphin propulsion etc. After that Mittra and Prasad [13] examined the influence of compliant walls on Poiseulli flow with peristalsis. They discussed the peristaltic flow in a two-dimensional channel. The relationship between stress and strain for viscous-inelastic fluids is discussed by Patel and Timol [14]. Heat transfer analysis has been used to obtain information about the properties of tissues and have many applications in the 
biomedical sciences with knowledge of initial thermal conditions. The influence of wall properties on peristaltic transport with heat transfer is presented by Radhakrishnamacharya and Srinivasulu [15]. Further Hayat et al. [16, 17] and Hina et al. [18] elaborated the concept of compliant walls with heat transfer for peristaltic transport. Rashidi et al. [19] examined the heat transfer effects in the flow by a non-isothermal wedge. Heat transfer with convective boundary conditions is involved in processes such as thermal energy storage, gas turbines, nuclear plants etc. Hayat et al. [20-22] presented the study of a non-Newtonian fluid with convective conditions. Recently many researchers discussed about the peristaltic transport of Prandtl fluid. Akbar et al. [23] examined the Prandtl fluid model in an asymmetric channel. Sucharitha et al. [24] and Navaneeswara et al. [25] discussed the conducting Prandtl fluid in a porous channel. Jothia et al. [26] further explained the peristaltic transport of same model under the effects of magnetic field.

The above mentioned studies show that no attempt has been made to investigate the heat transfer on the MHD peristaltic transport of Prandtl fluid with complaint walls and convective conditions. The presented research article is based on the consideration of such effects in peristaltic flows. The governing equations of Prandtl fluid model are simplified under the assumptions of long wavelength and low Reynolds number approximations and solved by using perturbation technique. The expressions for stream function, temperature and velocity are obtained. The effects of emerging parameters on the velocity and temperature rise are discussed physically and shown via graphs.

\section{Mathematical formulation}

We consider an incompressible MHD Prandtl fluid in a symmetric channel of width $2 d$. A uniform magnetic field $B_{0}$ is applied in the transverse direction to the flow. Consider the coordinate system (x, y) where $\mathrm{x}$-axis and $\mathrm{y}$-axis are taken parallel and transverse to the direction of wave propagation respectively. The flow is induced by periodic peristaltic waves of length $\lambda$ and amplitude $a$ with constant speed $c$ along the channel walls (see Fig. 1). The geometry of the wall is given by

$$
y= \pm \eta(x, t)= \pm\left[d+a \sin \frac{2 \pi}{\lambda}(x-c t)\right],
$$

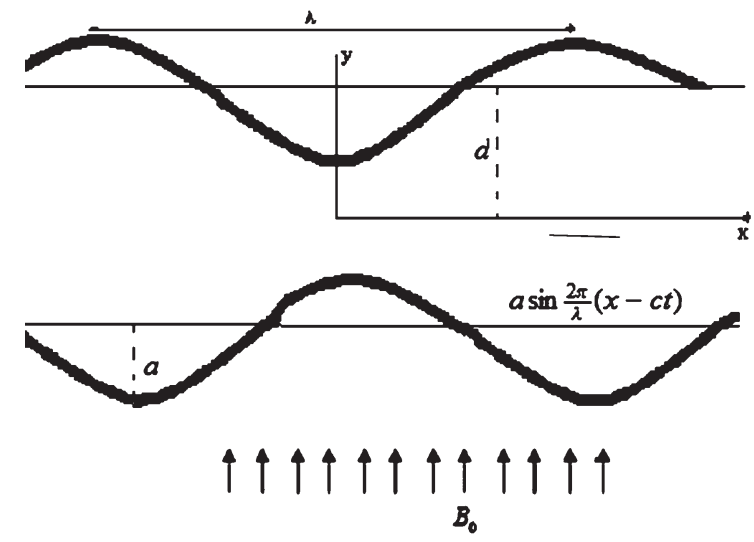

Fig. 1. Geometry of the problem.

The governing equations in the laboratory frame are

$$
\begin{gathered}
\frac{\partial u}{\partial x}+\frac{\partial v}{\partial y}=0, \\
\rho \frac{d u}{d t}=-\frac{\partial p}{\partial x}+\frac{\partial S_{x x}}{\partial x}+\frac{\partial S_{x y}}{\partial y}-\sigma B_{0}^{2} u, \\
\rho \frac{d v}{d t}=-\frac{\partial p}{\partial y}+\frac{\partial S_{y x}}{\partial x}+\frac{\partial S_{y y}}{\partial y}, \\
\rho C_{p} \frac{d T}{d t}=k\left[\frac{\partial^{2} T}{\partial x^{2}}+\frac{\partial^{2} T}{\partial y^{2}}\right]+\left(S_{x x}-S_{y y}\right) \frac{\partial u}{\partial x} \\
+S_{x y}\left(\frac{\partial v}{\partial x}+\frac{\partial u}{\partial y}\right),
\end{gathered}
$$

where $(u, v)$ are the components of velocity field $\mathbf{V}$ in the $x$ and $y$ directions respectively, $B_{0}$ is the strength of the constant applied magnetic field, $\sigma$ the electrical conductivity of fluid, $S_{i j}(i, j=x, y)$ the components of extra stress tensor, $d / d t$ the material time derivative, $C_{p}$ the specific heat at constant volume and $T$ the temperature of fluid. The extra stress tensor $S$ for Prandtl fluid is given by [14]

$$
S_{x y}=\frac{A \sin ^{-1}\left[\frac{1}{C}\left[\left(\frac{\partial u}{\partial y}\right)^{2}+\left(\frac{\partial v}{\partial x}\right)^{2}\right]^{1 / 2}\right]}{\left[\left(\frac{\partial u}{\partial y}\right)^{2}+\left(\frac{\partial v}{\partial x}\right)^{2}\right]^{1 / 2}} \frac{\partial u}{\partial y},
$$

in which $A$ and $C$ are material constants of Prandtl fluid model. 

by

The corresponding boundary conditions are given

$$
\begin{gathered}
u=0, \quad \text { at } y= \pm \eta \\
{\left[-\tau \frac{\partial^{3}}{\partial x^{3}}+m \frac{\partial^{3}}{\partial x \partial t^{2}}+d \frac{\partial^{2}}{\partial x \partial t}+B \frac{\partial^{5}}{\partial x^{5}}+H \frac{\partial}{\partial x}\right] \eta} \\
=+\frac{\partial S_{x x}}{\partial x}+\frac{\partial S_{x y}}{\partial y}-\sigma B_{0}^{2} u-\rho \frac{d u}{d t} \text { at } y= \pm \eta, \\
k \frac{\partial T}{\partial y}=-h_{1}\left(T-T_{0}\right) \quad \text { at } \quad y=\eta \\
k \frac{\partial T}{\partial y}=-h_{2}\left(T_{0}-T\right) \quad \text { at } \quad y=-\eta,
\end{gathered}
$$

In above equations $\tau$ is the elastic tension in the membrane, $m$ the mass per unit area, $d$ the coefficient of viscous damping, $B$ flexural rigidity of the plate, $H$ the spring stiffness. $h_{1}$ and $h_{2}$ the heat transfer coefficients and $T_{0}$ the ambient temperature at both upper and lower walls of the channel.

Defining velocity components $u$ and $v$ in terms of stream function and dimensionless variables as

$$
\begin{gathered}
u=\frac{\partial \psi}{\partial y}, v=-\frac{\partial \psi}{\partial x}, . \psi^{*}=\frac{\psi}{c d}, x^{*}=\frac{x}{\lambda}, \\
y^{*}=\frac{y}{d}, t^{*}=\frac{c t}{\lambda}, p^{*}=\frac{d^{2} p}{c \mu \lambda}, S_{i j}=\frac{d S_{i j}}{c \mu}, \\
\eta^{*}=\frac{\eta}{d}, h_{1}^{*}=\frac{h_{1}}{d}, h_{2}^{*}=\frac{h_{2}}{d}, \theta=\frac{T-T_{0}}{T_{0}},
\end{gathered}
$$

the Equation (2) is identically satisfied and Equations (3-10) in terms of stream function $\psi$ can be expressed as follows:

$$
\begin{array}{r}
\delta \operatorname{Re}\left[\frac{\partial}{\partial t}+\psi_{y} \frac{\partial}{\partial x}-\psi_{x} \frac{\partial}{\partial y}\right] \psi_{y} \\
=-\frac{\partial p}{\partial x}+\delta \frac{\partial S_{x x}}{\partial x}+\frac{\partial S_{x y}}{\partial y}-M^{2} \psi_{y}, \\
\delta^{3} \operatorname{Re}\left[\frac{\partial}{\partial t}+\psi_{y} \frac{\partial}{\partial x}-\psi_{x} \frac{\partial}{\partial y}\right] \psi_{x} \\
=-\frac{\partial p}{\partial y}+\delta^{2} \frac{\partial S_{x y}}{\partial x}+\delta \frac{\partial S_{y y}}{\partial y} \\
\delta \operatorname{Re} \operatorname{Pr}\left[\frac{\partial}{\partial t}+\psi_{y} \frac{\partial}{\partial x}-\psi_{x} \frac{\partial}{\partial y}\right]
\end{array}
$$

$$
\begin{gathered}
=\frac{\partial^{2} \theta}{\partial y^{2}}+\delta^{2} \frac{\partial^{2} \theta}{\partial x^{2}}+B r\left[\delta\left(S_{x x}-S_{y y}\right) \psi_{x y}\right. \\
\left.+S_{x y}\left(\psi_{y y}-\delta^{2} \psi_{x x}\right)\right] \\
\frac{\partial \psi}{\partial y}=0 \quad \text { at } \quad y= \pm \eta, \\
{\left[E_{1} \frac{\partial^{3}}{\partial x^{3}}+E_{2} \frac{\partial^{3}}{\partial x \partial t^{2}}+E_{3} \frac{\partial^{2}}{\partial x \partial t}+E_{4} \frac{\partial^{5}}{\partial x^{5}}+E_{5} \frac{\partial}{\partial x}\right] \eta} \\
=-M^{2} \psi_{y}-\delta \operatorname{Re}\left[\frac{\partial}{\partial t}+\psi_{y} \frac{\partial}{\partial x}-\psi_{x} \frac{\partial}{\partial y}\right] \psi_{y} \\
+\delta \frac{\partial S_{x x}}{\partial x}+\frac{\partial S_{x y}}{\partial y} \quad \text { at } \quad y= \pm \eta, \\
\frac{\partial \theta}{\partial y}+B i_{1} \theta=0 \quad \text { at } \quad y=+\eta, \\
\frac{\partial \theta}{\partial y}-B i_{2} \theta=0 \quad \text { at } \quad y=-\eta,
\end{gathered}
$$

where $\eta=(1+\epsilon \sin 2 \pi(x-t)), \epsilon=a / d$ the geometric parameter, $\delta=\frac{d}{\lambda}$ the wave number, $\operatorname{Re}=\frac{\rho c d}{\mu}$ the Reynolds number, $M=\sqrt{\sigma / \mu} B_{0} d$ the magnetic parameter, $B r=\operatorname{Pr} E c$ the Brinkman number, $\operatorname{Pr}=$ $\frac{\mu c_{p}}{k}$ and $E c=\frac{c^{2}}{T_{0} c_{p}}$ the Prandtl and Eckert numbers, $B i_{1}=h_{1} d / k$, and $B i_{2}=h_{2} d / k$ the Biot numbers, $E_{1}=-\tau d^{3} / \lambda^{3} \mu c, E_{2}=m c d^{3} / \lambda^{3} \mu, E_{3}=d^{4} / \lambda^{3} \mu$, $E_{4}=B d^{3} / \lambda^{3} c \mu$ and $E_{5}=H d^{3} / \lambda c \mu$ are the nondimensional elasticity parameters (here asterisks have been omitted for simplicity).

In the limit $\operatorname{Re} \rightarrow 0$, the inertialess flow corresponds to Poiseuille-like longitudinal velocity profile. The pressure gradient depends upon $x$ and $t$ only in laboratory frame. It does not depend on $y$. Such features can be expected because there is no streamline curvature to produce transverse pressure gradient when $\delta=0$. The assumptions of long wavelength and small Reynolds number give $\delta=0$ and $\operatorname{Re}=0$. It should be pointed out that the theory of long wavelength and zero Reynolds number remains applicable for case of chyme transport in small intestine [27]. In this case $c=2$ $\mathrm{cm} / \mathrm{min}, a=1.25 \mathrm{~cm}$ and $\lambda=8.01 \mathrm{~cm}$. Here half width of intestine is small in comparison to wavelength i.e. $a / \lambda=0.156$. It is also declared experimentally by Lew et al. [28] that Reynolds number in small intestine is small. Further, the situation of intrauterine fluid 
flow due to myomaterial contractions is a peristaltic type fluid motion in a cavity. The sagittal cross section of the uterus reveals a narrow channel enclosed by two fairly parallel walls [29]. The $1-3 \mathrm{~mm}$ width of this channel is very small compared with its $50 \mathrm{~mm}$ length [30], defining an opening angle from cervix to fundus of about $0.04 \mathrm{rad}$. Analysis of dynamics parameters of the uterus revealed frequency, wavelength, amplitude and velocity of the fluid-wall interface during a typical contractile wave were found to be $0.01-0.057$ $\mathrm{Hz}, 10-30 \mathrm{~mm}, 0.05-0.2 \mathrm{~mm}$ and $0.5-1.9 \mathrm{~mm} / \mathrm{s}$ respectively. Therefore adopting low Reynolds number and long wavelength analysis [1], Equations (12-18) reduce to the following forms:

$$
\begin{gathered}
\frac{\partial p}{\partial x}=\frac{\partial S_{x y}}{\partial y}-M^{2} \psi_{y} \\
\frac{\partial p}{\partial y}=0 \\
\frac{\partial^{2} \theta}{\partial y^{2}}+B r S_{x y} \psi_{y y}=0 \\
\psi_{y}=0 \quad \text { at } \quad y= \pm \eta \\
{\left[E_{1} \frac{\partial^{3}}{\partial x^{3}}+E_{2} \frac{\partial^{3}}{\partial x \partial t^{2}}+E_{3} \frac{\partial^{2}}{\partial x \partial t}+E_{4} \frac{\partial^{5}}{\partial x^{5}}+E_{5} \frac{\partial}{\partial x}\right] \eta} \\
=\frac{\partial S_{x y}}{\partial y}-M^{2} \psi_{y} \quad \text { at } y= \pm \eta, \\
\frac{\partial \theta}{\partial y}+B i_{1} \theta=0 \quad \text { at } \quad y=+\eta \\
\frac{\partial \theta}{\partial y}-B i_{2} \theta=0 \quad \text { at } \quad y=-\eta .
\end{gathered}
$$

From Equation (20), note that $p \neq p(y)$. Also the extra stress component from Equation (6) is given by

$$
S_{x y}=\alpha \psi_{y y}+\frac{\beta}{6}\left(\psi_{y y}\right)^{3},
$$

where $\alpha=\frac{A}{\mu C}, \beta=\frac{\alpha c^{2}}{C^{2} d^{2}}$.

The expression for heat transfer coefficient is given by

$$
Z=\eta_{x} \theta_{y}(\eta)
$$

\section{Solution methodology}

As the governing equations are highly nonlinear and exact solution seems to be impossible therefore perturbation method for small parameter $\beta$ is used to find the analytic solution. Thus we expand the flow quantities $\psi, S_{x y}, \theta$ and $Z$ as follows:

$$
\begin{gathered}
\psi=\psi_{0}+\beta \psi_{1}+O\left(\beta^{2}\right), \\
S_{x y}=S_{0 x y}+\beta S_{1 x y}+O\left(\beta^{2}\right), \\
\theta=\theta_{0}+\beta \theta_{1}+O\left(\beta^{2}\right), \\
Z=Z_{0}+\beta Z_{1}+O\left(\beta^{2}\right) .
\end{gathered}
$$

\subsection{Zeroth order system and its solution}

Using Equations (26-29) into Equations (19-25) and then collecting the coefficients of like powers of $\beta^{0}$, we get the zeroth order system as follows:

$$
\begin{gathered}
\frac{\partial^{4} \psi_{0}}{\partial y^{4}}-\frac{M^{2}}{\alpha} \frac{\partial^{2} \psi_{0}}{\partial y^{2}}=0 \\
\frac{\partial^{2} \theta_{0}}{\partial y^{2}}+\alpha B r\left(\frac{\partial^{2} \psi_{0}}{\partial y^{2}}\right)^{2}=0 \\
\frac{\partial \psi_{0}}{\partial y}=0, \quad \text { at } y= \pm \eta \\
\left.E_{1} \frac{\partial^{3}}{\partial x^{3}}+E_{2} \frac{\partial^{3}}{\partial x \partial t^{2}}+E_{3} \frac{\partial^{2}}{\partial x \partial t}+E_{4} \frac{\partial^{5}}{\partial x^{5}}+E_{5} \frac{\partial}{\partial x}\right] \eta \\
=\frac{\partial}{\partial y}\left[\frac{\partial^{2} \psi_{0}}{\partial y^{2}}\right]-M^{2} \frac{\partial \psi_{0}}{\partial y} \quad \text { at } y= \pm \eta \\
\frac{\partial \theta_{0}}{\partial y}+B i_{1} \theta_{0}=0 \quad \text { at } \quad y=+\eta \\
\frac{\partial \theta_{0}}{\partial y}-B i_{2} \theta_{0}=0 \quad \text { at } \quad y=-\eta
\end{gathered}
$$

The solutions of Equations (30-35) are given by

$$
\psi_{0}=A_{1} y+A_{2} \sin h\left[\frac{M y}{\sqrt{\alpha}}\right]
$$

$$
\theta_{0}=L_{1} y+L_{2} y^{2}+L_{3} \cosh \left[\frac{2 M y}{\sqrt{\alpha}}\right]+L_{4},
$$

Heat transfer coefficient is 


$$
\begin{aligned}
Z_{0}= & \eta_{x} \theta_{0 y}(\eta) \\
= & \frac{\eta_{x} B i_{1}\left(1+\eta B i_{2}\right)}{M^{3}\left(B i_{2}+B i_{1}\left(1+2 \eta B i_{2}\right)\right)} \\
& \left(2 A_{0}^{2} \sqrt{B_{0}} B r \pi^{2} \varepsilon^{2}\left(2 M \eta-\sqrt{\alpha} \sin h\left(\frac{2 M \eta}{\sqrt{\alpha}}\right)\right) .\right.
\end{aligned}
$$

\subsection{First order system and its solution}

The coefficients of likes powers of $\beta$ yield the following system of equations:

$$
\begin{gathered}
\alpha \frac{\partial^{4} \psi_{1}}{\partial y^{4}}-M^{2} \frac{\partial^{2} \psi_{1}}{\partial y^{2}}+\frac{\partial^{2} \psi_{0}}{\partial y^{2}}\left(\frac{\partial^{3} \psi_{0}}{\partial y^{3}}\right)^{2} \\
+\frac{\partial^{4} \psi_{0}}{\partial y^{4}}\left(\frac{\partial^{2} \psi_{0}}{\partial y^{2}}\right)^{2}=0 \\
\frac{\partial^{2} \theta_{1}}{\partial y^{2}}+B r\left(\alpha\left(\frac{\partial^{2} \psi_{0}}{\partial y^{2}} \frac{\partial^{2} \psi_{1}}{\partial y^{2}}\right)^{2}\right. \\
\left.\quad+\frac{1}{6}\left(\frac{\partial^{2} \psi_{0}}{\partial y^{2}}\right)^{4}\right)=0 \\
\frac{\partial \psi_{1}}{\partial y}=0, \quad \text { at } y= \pm \eta \\
\alpha \frac{\partial^{3} \psi_{1}}{\partial y^{3}}-M^{2} \frac{\partial \psi_{1}}{\partial y}+\frac{1}{2} \frac{\partial^{3} \psi_{0}}{\partial y^{3}}\left(\frac{\partial^{2} \psi_{0}}{\partial y^{2}}\right)^{2}=0 \\
\text { at } y= \pm \eta, \\
\frac{\partial \theta_{1}}{\partial y}+B i_{1} \theta_{1}=0 \quad \text { at } \quad y=+\eta \\
\frac{\partial \theta_{1}}{\partial y}-B i_{2} \theta_{1}=0
\end{gathered}
$$

Invoking the zeroth order solution into the first order system and then solving the resulting system we have

$$
\begin{aligned}
\psi_{1}= & A_{0}^{3} B_{0} \pi^{3} \varepsilon^{3}\left[3\left(9+8 B_{1}-B_{2}\right) M y\right. \\
& +36 B_{3} M y \cos h(M y / \sqrt{\alpha}) \\
& +9\left(4 B_{5} M+\left(-10 B_{3}+B_{4}\right) \sqrt{\alpha}\right) \sinh (M y / \sqrt{\alpha}) \\
& \left.-B_{3} \sqrt{\alpha} \sinh (M y / \sqrt{\alpha})\right], \\
\theta_{1}= & D_{11} y^{4}+y\left(D_{7} \sinh (2 M y / \sqrt{\alpha})+D_{0}\left(D_{10}\right.\right. \\
& \left.\left.-D_{8} \sinh (4 M y / \sqrt{\alpha})+D_{9} \sinh (6 M y / \sqrt{\alpha})\right)\right)
\end{aligned}
$$

$$
\begin{aligned}
+ & D_{0}\left(D_{2}+\alpha\left(D_{3} \cosh (2 M y / \sqrt{\alpha})\right.\right. \\
- & D_{4} \cosh (4 M y / \sqrt{\alpha})+D_{5} \cosh (6 M y / \sqrt{\alpha}) \\
- & \left.D_{6} \cosh (8 M y / \sqrt{\alpha})+D_{6} \cosh (8 M y / \sqrt{\alpha})\right) \\
& \left.\left(B i_{2}+B i_{1}\left(1+2 \eta B i_{2}\right)\right)\right)+y^{2} \cosh (4 M y / \sqrt{\alpha}) \\
\times & \left(D_{12}-D_{13} \cosh (4 M y / \sqrt{\alpha})\right),
\end{aligned}
$$

$$
\begin{aligned}
Z_{1}= & \eta_{x} \theta_{1 y}(\eta) \\
= & \eta_{x}\left(4 D_{11} \eta^{3}+\left(2 M \eta \left(D_{7} \cosh (2 M \eta / \sqrt{\alpha})\right.\right.\right. \\
& -2 D_{0} D_{8} \cosh (4 M \eta / \sqrt{\alpha})+3 D_{0} D_{9} \cosh (6 M \eta \\
& / \sqrt{\alpha}))) / \sqrt{\alpha}+D_{7} \sinh (M \eta / \sqrt{\alpha}) \\
& +D_{0}\left(D_{10}-D_{8} \sinh (4 M \eta / \sqrt{\alpha})\right. \\
& \left.+D_{9} \sinh (6 M \eta / \sqrt{\alpha})\right) \\
& +2 D_{0} M \sqrt{\alpha}\left(D_{3} \sinh (2 M \eta / \sqrt{\alpha})\right. \\
& -2 D_{4} \sinh (4 M \eta / \sqrt{\alpha})+3 D_{5} \sinh (6 M \eta / \sqrt{\alpha}) \\
& \left.-4 D_{6} \sinh (8 M \eta / \sqrt{\alpha})\right)\left(B i_{2}+B i_{1}\left(1+2 \eta B i_{2}\right)\right) \\
& +2 \eta \cosh (4 M \eta / \sqrt{\alpha}) \times\left(D_{12}-D_{13} \cosh (4 M \eta\right. \\
& / \sqrt{\alpha}))+\left(\eta^{2} / \sqrt{\alpha}\right)(4 M \sinh (4 M \eta / \sqrt{\alpha}) \\
& \times\left(D_{12}-D_{13} \cosh (4 M \eta / \sqrt{\alpha})\right) \\
& -(1 / \sqrt{\alpha}) 4 D_{13} M \sinh (4 M \eta / \sqrt{\alpha}) \cosh (4 M \eta \\
& \left./ \sqrt{\alpha}) \times\left(D_{12}-D_{13} \cosh (4 M \eta / \sqrt{\alpha})\right)\right)
\end{aligned}
$$

The constants $A_{i}^{\prime} s(i=0-2), B_{j} / s(j=0-3)$, $D_{k} s(k=0-13)$ and $L_{m}^{\prime} s(m=1,4)$ are given in Appendix A.

\section{Graphical results and discussion}

Figs. (1-7) are presented to observe the behavior of emerging parameters involved in the solution expressions of longitudinal velocity $\left(u=\psi_{0 y}+\beta \psi_{1 y}\right)$, temperature $\theta$, heat transfer coefficient $Z$ and stream function $\psi$.

\subsection{Velocity profile}

Velocity profile is plotted in Fig. 2 to study the effects of various values of Hartman number $(M)$, Fluid parameters $(\alpha$ and $\beta)$ and wall parameters $\left(E_{1}, E_{2}\right.$, $E_{3}, E_{4}, E_{5}$ ). Fig. (2a) shows that the velocity decreases with an increase in the fluid parameter $\alpha$. While Prandtl 

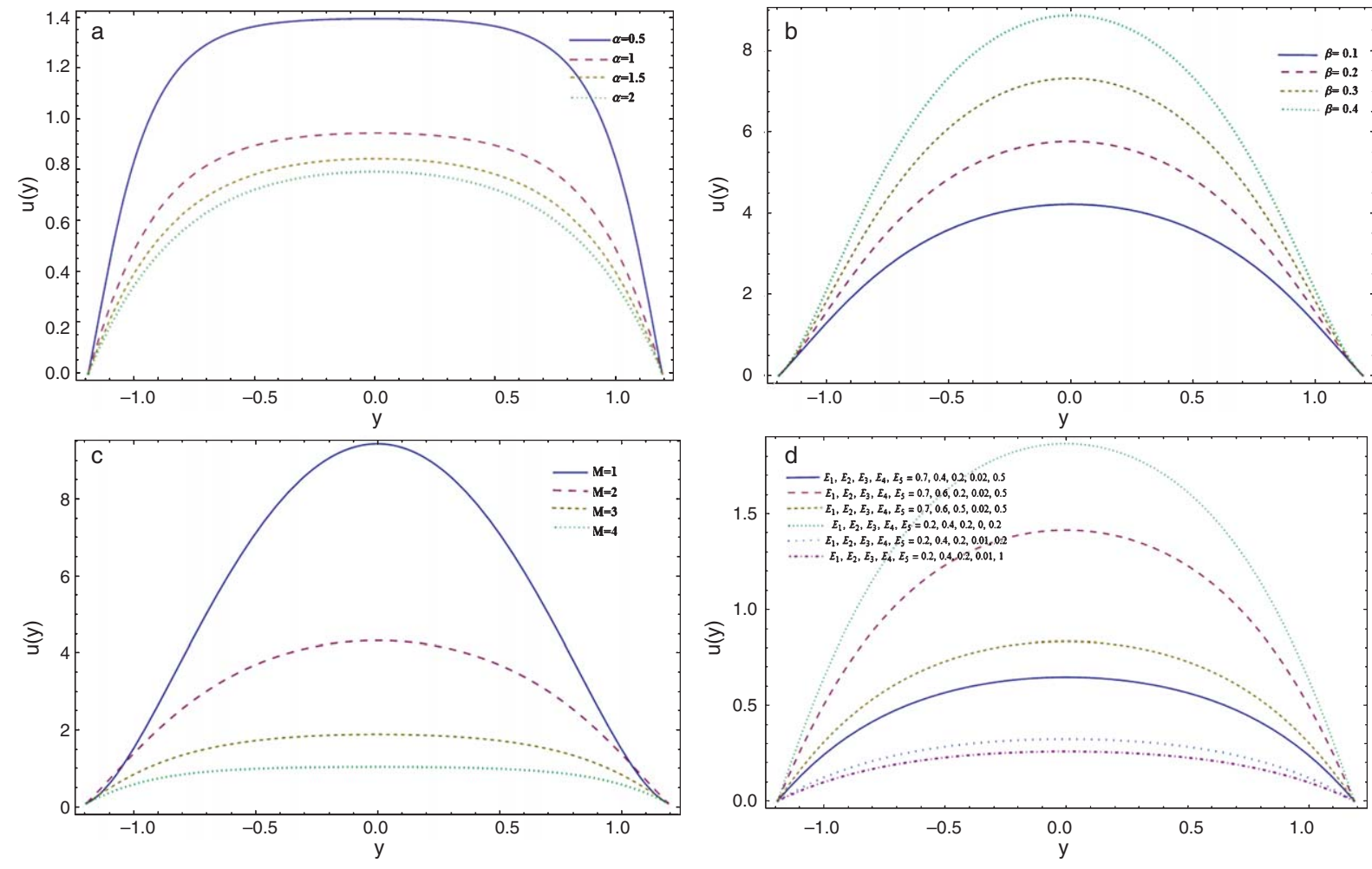

Fig. 2. (a): Variation of $\alpha$ on $u$ when $E_{1}=1, E_{2}=0.5, E_{3}=0.5, E_{4}=0.01, E_{5}=0.3, \epsilon=0.2, x=0.3, t=0.1, M=4$ and $\beta=0.1$. (b) Variation of $\beta$ on $u$ when $E_{1}=1, E_{2}=0.5, E_{3}=0.5, E_{4}=0.01, E_{5}=0.3, \epsilon=0.2, x=0.3, t=0.1, M=2$ and $\alpha=1$. (c): Variation of $M$ on $u$ when $E_{1}=1, E_{2}=0.5, E_{3}=0.5, E_{4}=0.01, E_{5}=0.3, \epsilon=0.2, x=0.3, t=0.1, \beta=0.1$ and $\alpha=1$. (d): Variation of $E_{1}, E_{2}$, $E_{3}, E_{4}, E_{5}$ on $u$ when $M=2, \epsilon=0.2, x=0.3, t=0.1, \beta=0.1$ and $\alpha=1$.

fluid parameter $\beta$ has opposite effect on velocity profile i.e. velocity increases with increase in Prandtl fluid parameter $\beta$ (see Fig. (2b)). Fig. (2c) depicts that the velocity profile decreases with an increase in the Hartman number $M$, because when the magnetic field $B_{0}$ is applied in transverse direction, it gives a resistance to the flow. Fig. (2d) depicts that with an increase in the mass per unit area $E_{2}$ and the coefficient of viscous damping $E_{3}$ the velocity increases and it decreases when the elastic tension in the membrane $E_{1}$ the flexural rigidity of the plate $E_{4}$ and the spring stiffness $E_{5}$ are increased. It is also worth to highlight that when the parameters are assigned a fixed value the velocity profile is parabolic and has maximum magnitude near the center of the channel.

\subsection{Temperature profile}

Figure 3 represents $\theta(y)$ for different values of $B i_{1}$, $B i_{2}, M, E_{1}, E_{2}, E_{3}, E_{4}, E_{5}, \alpha$ and $\beta$. Fig. (3a) illustrates that the temperature decreases with an increase in the fluid parameters $\alpha$. While temperature has opposite effect for the Prandtl fluid parameter $\beta$. Temperature increases with increase in Prandtl fluid parameter $\beta$ (see Fig. (3b)). Fig. (3c) shows that by increasing the $B i_{1}$ the temperature decreases near the upper wall of channel but it has no effect near lower wall of channel. Similarly Fig. (3d) reveals that by increasing $B i_{2}$ the temperature profile decreases near lower wall of channel and it has no meaningful effect near upper wall of the channel. Fig. (3e) depicts that $\theta$ decreases by increasing $M$. The effects of compliant wall parameters in Fig. (3f) indicates that by increasing $E_{1}$ and $E_{2}$, the temperature enhances whereas by increasing values of $E_{3}, E_{4}$ and $E_{5}$ temperature is decaying.

\subsection{Heat transfer coefficient}

In Fig. 4 we observed the variation in heat transfer coefficient $Z(x)$ for different parameters $B i_{1}, B i_{2}, M$, $E_{1}, E_{2}, E_{3}, E_{4}, E_{5}, \alpha$ and $\beta$ appeared in the solution. The nature of the heat transfer coefficient is oscilla- 

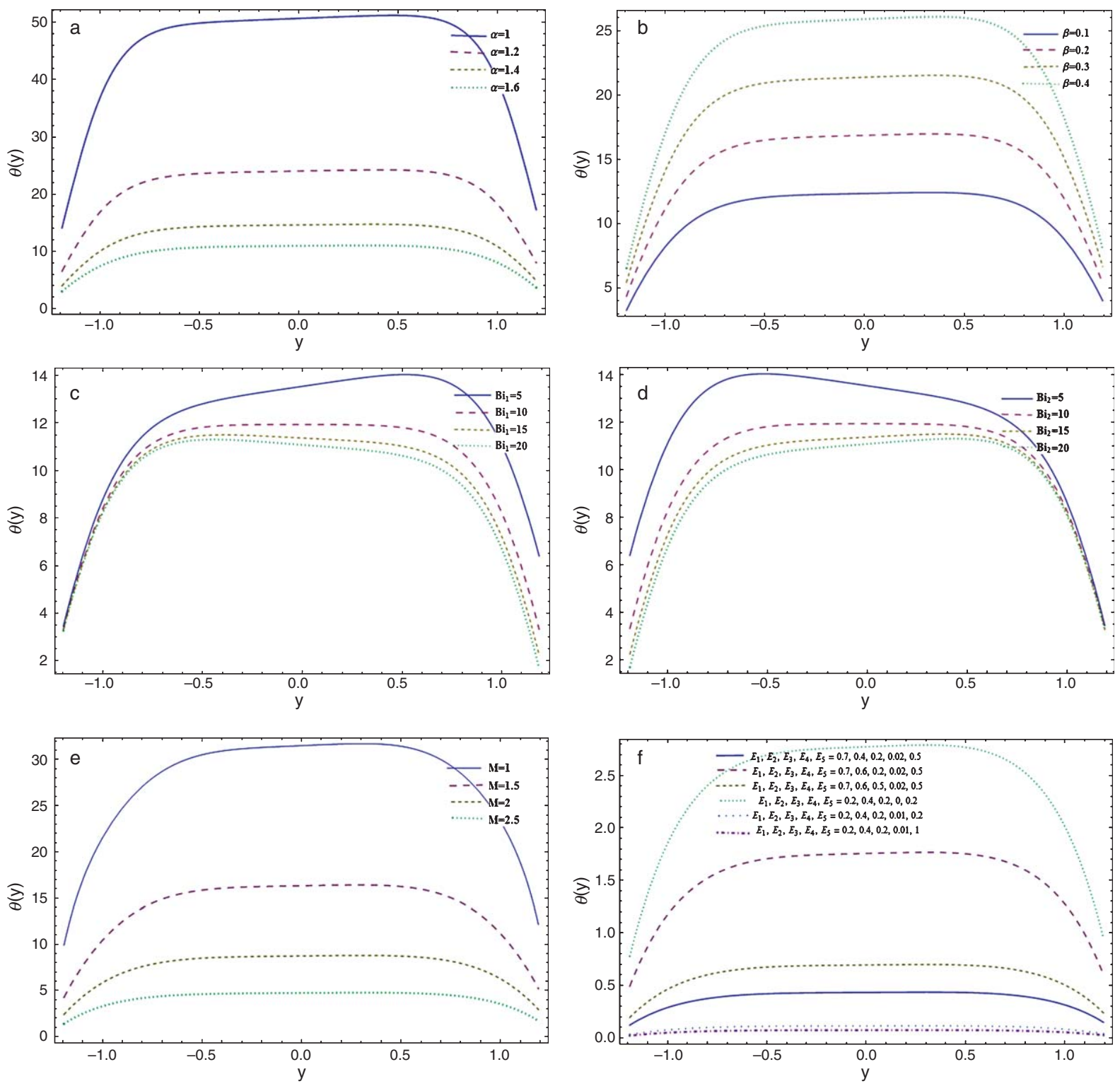

Fig. 3. (a): Variation of $\alpha$ on $\theta$ when $E_{1}=1, E_{2}=E_{3}=0.5, E_{4}=0.01, E_{5}=0.3, \epsilon=0.2, x=0.3, t=0.1, B r=M=2, \beta=0.02$ and $B i_{2}=10$. (b): Variation of $\beta$ on $\theta$ when $E_{1}=1, E_{2}=E_{3}=0.5, E_{4}=0.01, E_{5}=0.3, \epsilon=0.2, x=0.3, t=0.1, \alpha=B r=M=2$ and $B i_{1}=8$. (c): Variation of $B i_{1}$ on $\theta$ when $E_{1}=1, E_{2}=E_{3}=0.5, E_{4}=0.01, E_{5}=0.3, \epsilon=0.2, x=0.3, t=0.1, \alpha=1.5, \beta=0.02$, $B r=M=2$ and $B i_{2}=10$. (d): Variation of $B i_{2}$ on $\theta$ when $E_{1}=1, E_{2}=E_{3}=0.5, E_{4}=0.01, E_{5}=0.3, \epsilon=0.2, x=0.3, t=0.1, \alpha=1.5$, $\beta=0.02, B r=M=2$ and $B i_{1}=8$. (e): Variation of $M$ on $\theta$ when $E_{1}=1, E_{2}=E_{3}=0.5, E_{4}=0.01, E_{5}=0.3, \epsilon=0.2, x=0.3, t=0.1$, $\alpha=2, \beta=0.02, B r=2, B i_{1}=8$ and $B i_{2}=10$. (f): Variation of compliant wall parameters on $\theta$ when $\epsilon=0.2, x=0.3, t=0.1, B i_{1}=8$, $\alpha=2, \beta=0.02$ and $B i_{2}=10$.

tory in nature due to peristalsis motion of the waves. Magnitude of heat transfer coefficient $Z(x)$ has increasing behavior for different values of $\alpha$ (see Fig. (4a)). For increasing $\beta$, magnitude of heat transfer coefficient $Z(x)$ also increases (see Fig. (4b)). Further for increasing $B i_{1}$ the magnitude of heat transfer coeffi- cient $Z(x)$ increases and it decreases for $B i_{2}$ (see Figs. (4c) and (4d)). Magnitude of heat transfer coefficient $Z(x)$ decreases when Hartman number $M$ is increased (see Fig. (4e)). Fig. (4f) depicts that magnitude of heat transfer coefficient $Z(x)$ increases with the increase in $E_{1}, E_{2}$ and $E_{3}$ and it decreases for $E_{4}$ and $E_{5}$. 

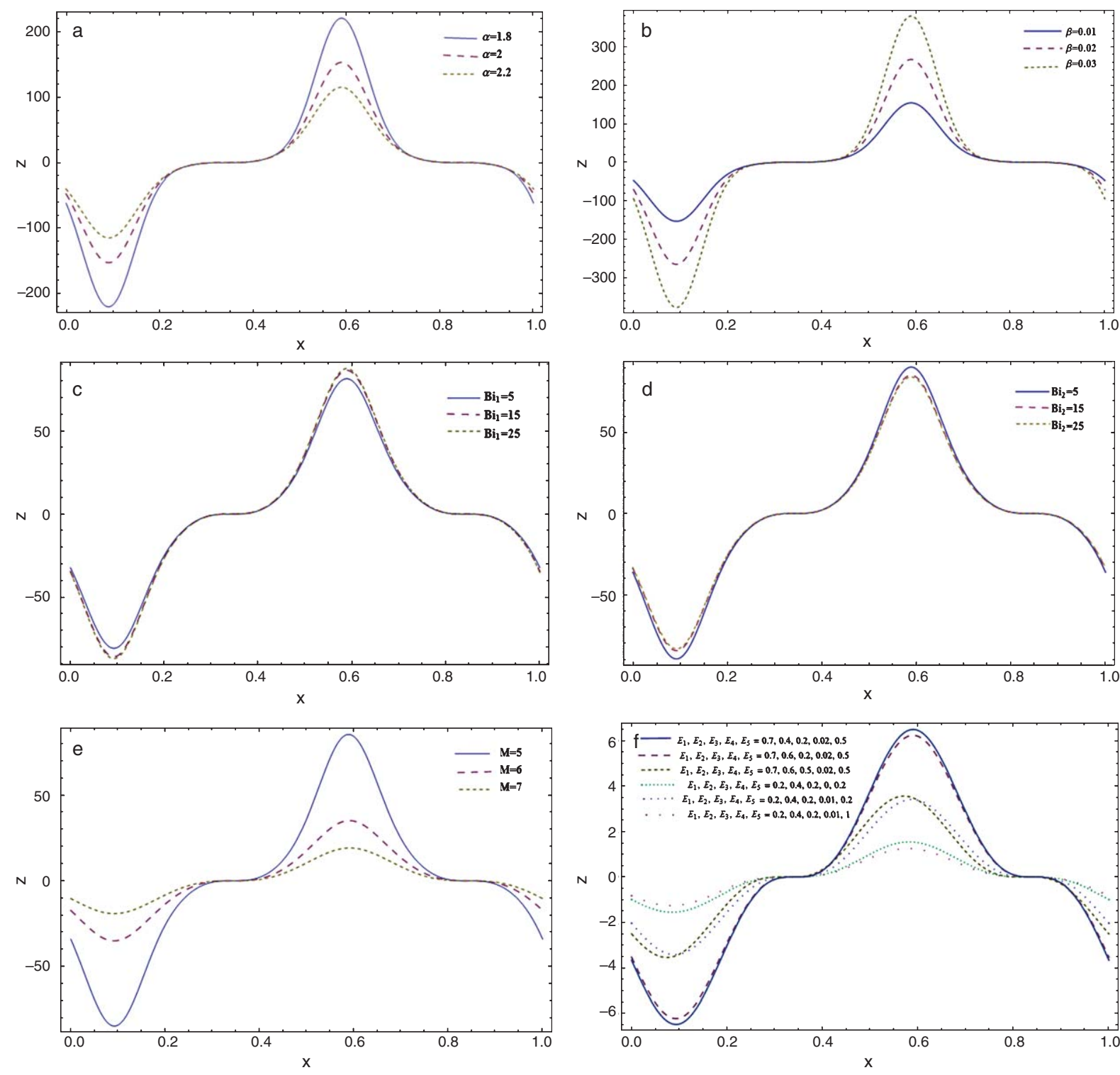

Fig. 4. (a): Variation of $\alpha$ on $Z$ when $M=5, E_{1}=1, E_{2}=E_{3}=0.5, E_{4}=0.01, E_{5}=0.3, \epsilon=0.2, t=0.1, \beta=0.01, B r=2, B i_{1}=8$ and $B i_{2}=10$. (b): Variation of $\beta$ on $Z$ when $M=5, E_{1}=1, E_{2}=E_{3}=0.5, E_{4}=0.01, E_{5}=0.3, \epsilon=0.2, t=0.1, \alpha=2, B r=2, B i_{1}=8$ and $B i_{2}=10$. (c): Variation of $B i_{1}$ on $Z$ when $M=5, E_{1}=1, E_{2}=E_{3}=0.5, E_{4}=0.01, E_{5}=0.3, \epsilon=0.2, t=0.1, \beta=0.01, \alpha=2.5, B r=2$ and $B i_{2}=15$. (d): Variation of $B i_{2}$ on $Z$ when $M=5, E_{1}=1, E_{2}=E_{3}=0.5, E_{4}=0.01, E_{5}=0.3, \epsilon=0.2, t=0.1, \beta=0.01, B r=2$ and $B i_{1}=10$. (e): Variation of $M$ on $Z$ when $E_{1}=1, E_{2}=E_{3}=0.5, E_{4}=0.01, E_{5}=0.3, \epsilon=0.2, t=0.1, \beta=0.01, \alpha=2.5, B r=2$, $B i_{1}=8$ and $B i_{2}=10$. (f): Variation of parameters of wall properties on $Z$ when $M=5, \epsilon=0.2, x=0.3, t=0.1, \beta=0.01, B r=2, \alpha=2.5$, $B i_{1}=8$ and $B i_{2}=10$.

\subsection{Trapping}

The formulation of an internally circulating bolus of fluid by closed streamlines is called trapping. We observed in Fig. $5(\mathrm{a}-\mathrm{c})$ that the size of trapped bolus increases with increase in Hartman number M. Fig. 6 (a-c) depicts that the size of trapped bolus increases when we increase the values of fluid parameter $\alpha$. It is also observed that the number of streamlines increases too. Fig. $7(\mathrm{a}-\mathrm{c})$ shows that the size of trapped bolus increases when we increase the values of fluid parameter $\beta$. We analyzed that the size of trapped bolus 

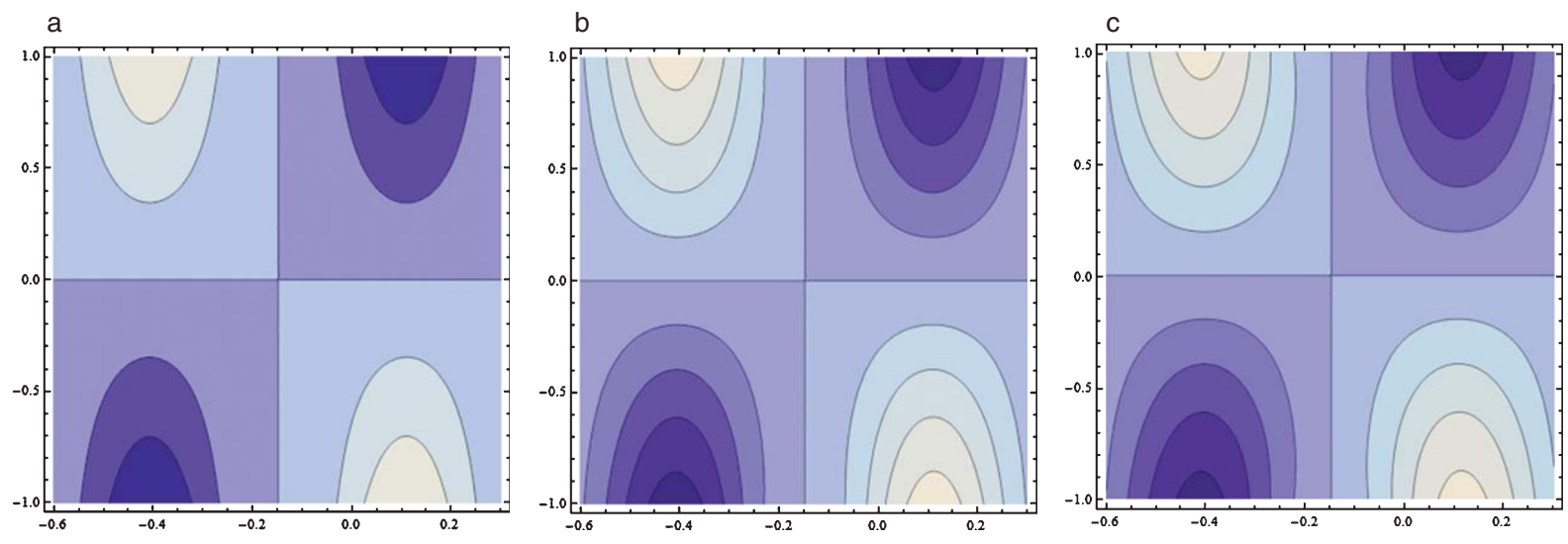

Fig. 5. Variation of $M$ on $\psi$ for $E_{1}=0.5, E_{2}=0.2, E_{3}=0.1, E_{4}=0.05, E_{5}=0.3, \epsilon=0.2, t=0.1, \alpha=1.5, \beta=0.02$, when (a): $M=2$ (b): $M=3$ (c): $M=4$.
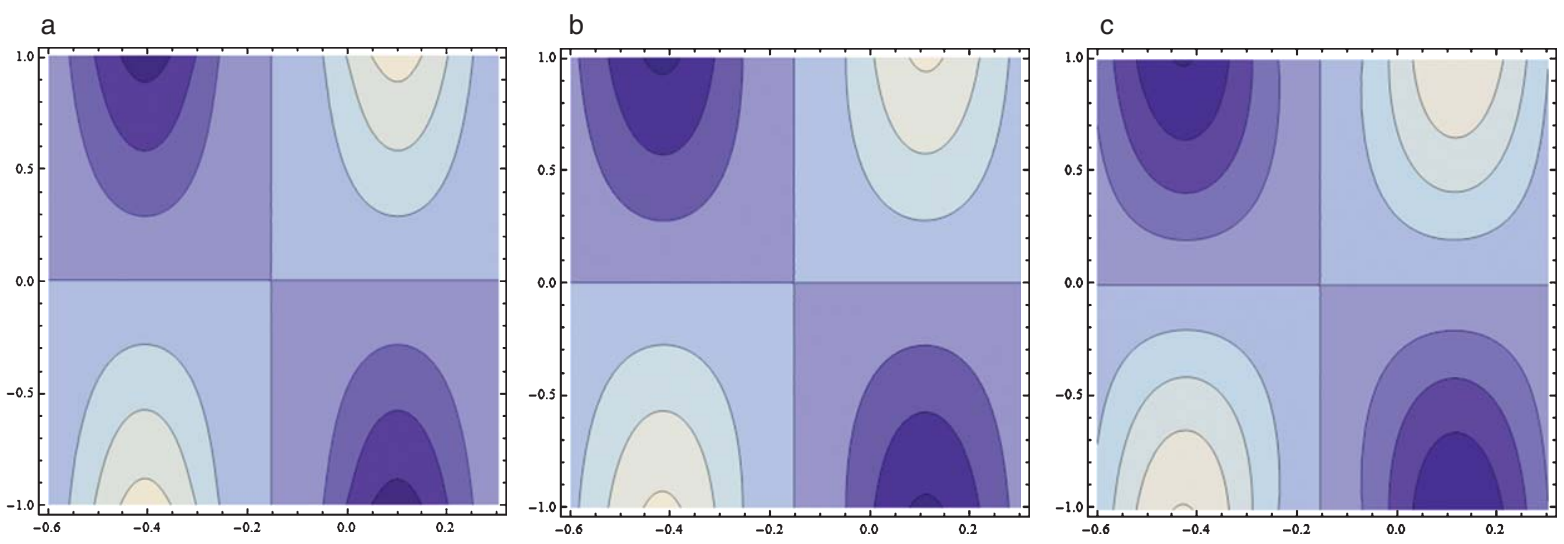

Fig. 6. Variation of $\alpha$ on $\psi$ for $E_{1}=0.7, E_{2}=0.2, E_{3}=0.1, E_{4}=0.01, E_{5}=0.3, \epsilon=0.2, M=2, t=0.1, \beta=0.02$, when (a): $\alpha=0.5$, (b): $\alpha=1,(\mathrm{c}): \alpha=1.5$.

a

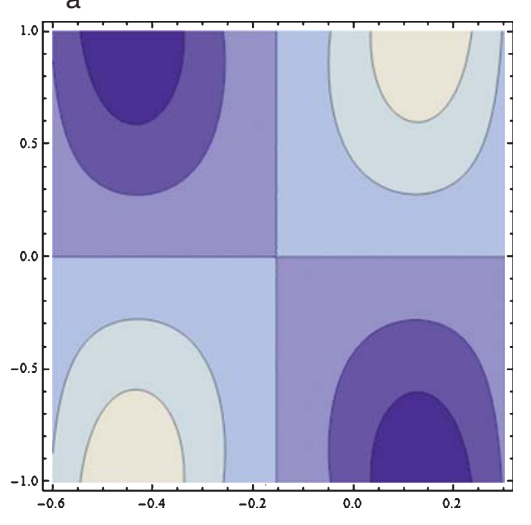

b

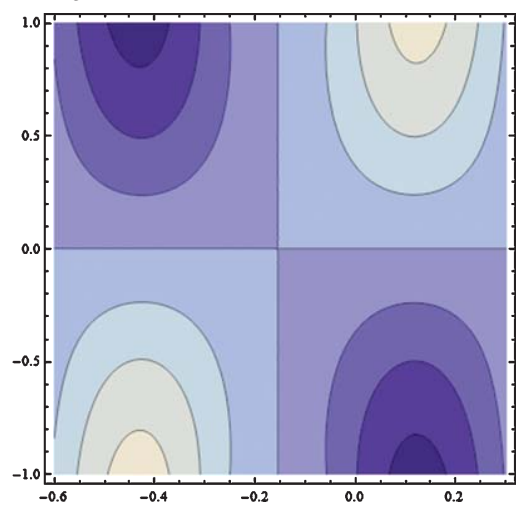

C

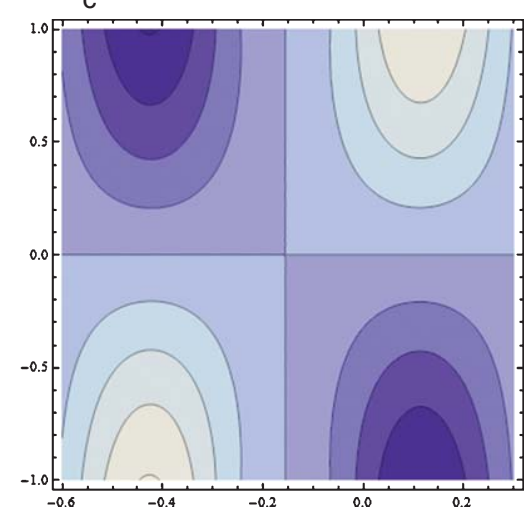

Fig. 7. Variation of $\beta$ on $\psi$ for $E_{1}=0.7, E_{2}=0.2, E_{3}=0.1, E_{4}=0.01, E_{5}=0.3, \epsilon=0.2, M=2, t=0.1, \alpha=2$, when (a): $\beta=0.01$, (b): $\beta=0.03$, (c): $\beta=0.05$. 
a

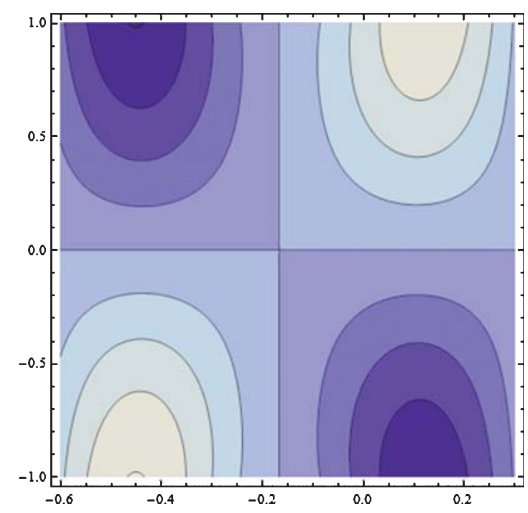

d

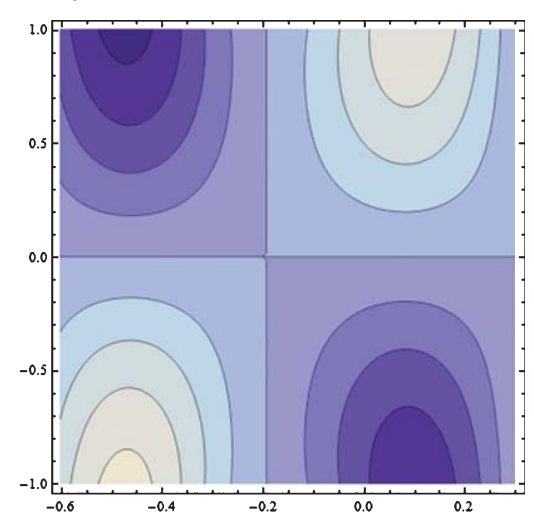

b
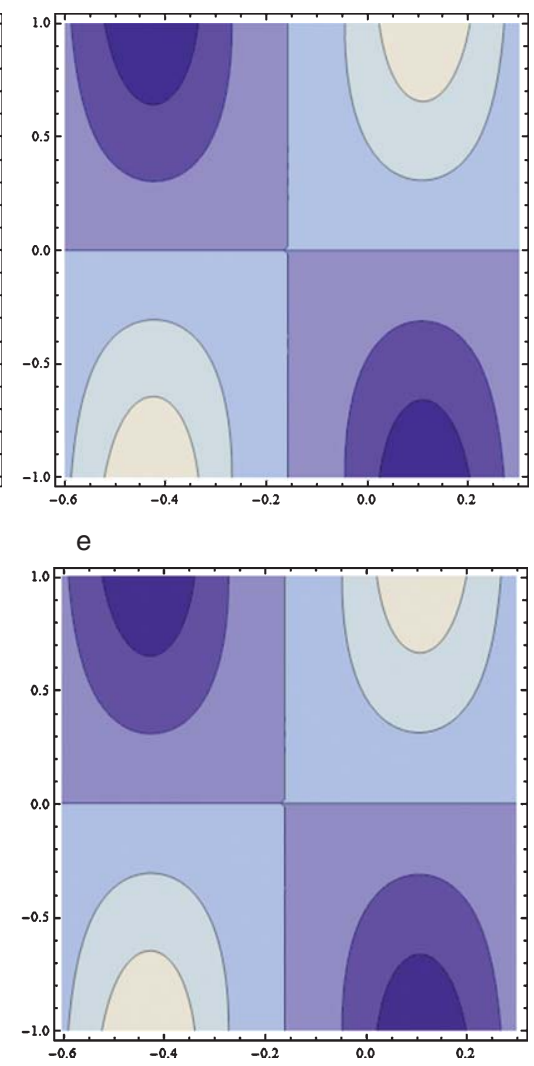

C

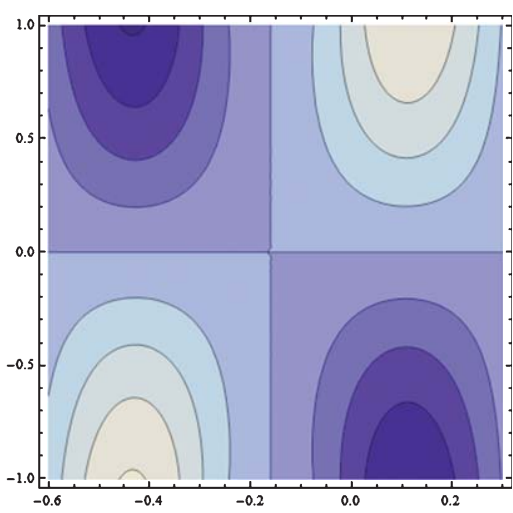

$f$

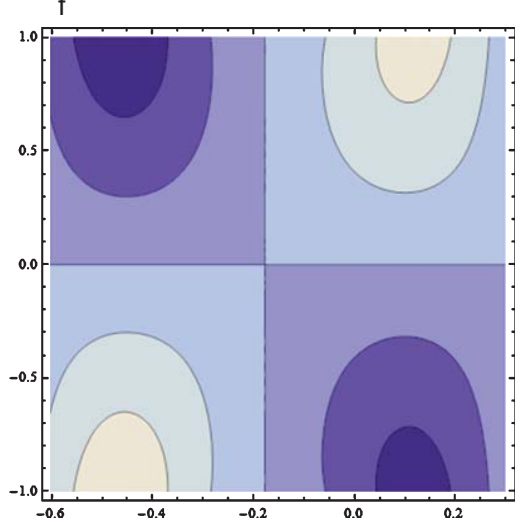

Fig. 8. Variation of wall properties on $\psi$ for $M=2, t=1, \beta=0.02, \alpha=1.5, \epsilon=0.2$, when (a): $E_{1}=0.7, E_{2}=0.4, E_{3}=0.2, E_{4}=0.02$, $E_{5}=0.5$, (b): $E_{1}=1, E_{2}=0.4, E_{3}=0.2, E_{4}=0.02, E_{5}=0.5,(\mathrm{c}): E_{1}=0.7, E_{2}=0.6, E_{3}=0.2, E_{4}=0.02, E_{5}=0.5,(\mathrm{~d}): E_{1}=0.7$, $E_{2}=0.4, E_{3}=0.5, E_{4}=0.02, E_{5}=0.5$, (e): $E_{1}=0.2, E_{2}=0.4, E_{3}=0.2, E_{4}=0, E_{5}=0.2,(\mathrm{f}): E_{1}=0.2, E_{2}=0.4, E_{3}=0.2$, $E_{4}=0.01, E_{5}=0.2$.

increases with an increase in the mass per unit area $E_{2}$ and the coefficient of viscous damping $E_{3}$. However it decreases when the elastic tension in the membrane $E_{1}$ is increased. Also when we decrease the values of the flexural rigidity of the plate $E_{4}$ and the spring stiffness $E_{5}$ the trapped bolus size decreases (see Fig. 8 (a-f)).

\section{Concluding remarks}

In the conducted study peristaltic transport of Prandtl fluid in a symmetric channel with convective conditions is examined in the presence of complaint wall properties. The main points of the above analysis are as follows:

- Prandtl fluid parameters $\alpha$ and $\beta$ have opposite effects on velocity and temperature profiles and heat transfer coefficient.
- The velocity profile has decreasing behavior for increasing values of Hartman number $M$.

- Temperature profile is a decreasing function of Biot numbers $B i_{1}$ and $B i_{2}$.

- Heat transfer coefficient $Z(x)$ increases for $B i_{1}$ and it decreases when $B i_{2}$ is increased.

- Size of trapped bolus increases for both fluid parameters $\alpha$ and $\beta$.

\section{References}

[1] A.H. Shapiro, M.Y. Jaffrin and S.L. Weinberg, Peristaltic pumping with long wave-lengths at low reynolds number, Journal of Fluid Mechanics 37 (1969), 799-825.

[2] Kh.S. Mekheimer, Effect of induced magnetic field on peristaltic flow of a couple stress fluid, Physics Letters A 372 (2008), 4271-4278.

[3] A. Ebaid, Effects of magnetic field and wall slip conditions on the peristaltic transport of a Newtonian fluid in an asymmetric channel, Physics Letters A 372 (2008), 4493-4499. 
[4] T. Hayat, Y. Wang, K. Hutter, S. Asghar and A.M. Siddiqui, Peristaltic transport of an Oldroyd-B fluid in a planar channel, Mathematical Problems in Engineering 4 (2004), 347-376.

[5] A. Medhavi, Peristaltic pumping of a non-newtonian fluid, Applications and Applied Mathematics 3 (2008), 137-148.

[6] M. Keimanesha, M.M. Rashidi, Ali J. Chamkha and R. Jafari, Study of a third grade non-Newtonian fluid flow between two parallel plates using the multi-step differential transform method, Computers \& Mathematics with Applications 62 (2011), 2871-2891.

[7] T. Hayat, M. Javed and S. Asghar, MHD peristaltic motion of Johnson-Segalman fluid in a channel with compliant walls, Physics Letters A 372 (2008), 5026-5036.

[8] S. Srinivas, R. Gayathri and M. Kothandapani, The influence of slip conditions, wall properties and heat transfer on MHD peristaltic transport, Computer Physics Communications 180 (2009), 2115-2122.

[9] P.R. Sharma and I.K. Dadheech, Effect of joule heating on steady MHD flow of low prandtl fluid on a porous stretching sheet, International Journal of Engineering Research and Technology 1 (2012), ISSN: 2278-0181.

[10] S. Noreen and M. Qasim, Peristaltic flow of MHD eyringpowell fluid in a channel, European Physical Journal Plus 128 (2013), 91-100.

[11] O. Anwar Bég, M.M. Rashidi, T.A. Bég and M. Asadi, Homotopy analysis of transient magneto-bio-fluid dynamics of micropolar squeeze film in a porous medium: A model for magneto-bio-rheological lubrication, Journal of Mechanics in Medicine and Biology 12 (2012), 1250051 (21 pages).

[12] M.O. Kramer, Readers forum, Journal of the Aerospace Sciences 68 (1960).

[13] T.K. Mittra and S.N. Prasad, On the influence of wall properties and poiseuille flow in peristalsis, Journal of Biomechanics 6 (1973), 681-693.

[14] M. Patel and M.G. Timol, The stress strain relationship for viscous-inelastic non-Newtonian fluids, International Journal of Applied Mathematics and Mechanics 6 (2010), 79-93.

[15] G. Radhakrishnamacharya and Ch. Srinisvasulu, Influence of wall properties on peristaltic transport with heat transfer, Comptes Rendus Mécanique 335 (2007), 369-373.

[16] T. Hayat, M. Javid and A.A Hendi, Peristaltic transport of viscous fluid in a curved channel with compliant walls, International Journal of Heat and Mass Transfer 54 (2011), 1615-1621.

[17] T. Hayat and S. Hina, The influence of wall properties on the MHD peristaltic flow of a Maxwell fluid with heat and mass transfer, Nonlinear Analysis: Real World Applications 11 (2010), 3155-3169.

[18] S. Hina, T. Hayat, S. Asghar and Awatif A. Hendi, Influence of compliant walls on peristaltic motion with heat/mass transfer and chemical reaction, International Journal of Heat and Mass Transfer 55 (2012), 3386-3394.

[19] M.M. Rashidi, M.T. Rastegari, M. Asadi and O. Anwar Bég, A study of non-Newtonian flow and heat transfer over a non-isothermal wedge using the homotopy analysis method, Chemical Engineering Communications 199 (2012), DOI:10.1080/00986445.2011.586756

[20] T. Hayat, H. Yasmin, M.S. Alhuthali and M.A. Kutbi, Peristaltic flow of a non-Newtonian fluid in an asymmetric channel with convective boundary conditions, Journal of Mechanics 29 (2013), 599-607.
[21] T. Hayat, H. Yasmin, B. Ahmed and B. Chen, Simultaneous effects of convective conditions and nanoparticles on peristaltic motion, Journal of Molecular Liquids 193 (2014), 74-82.

[22] T. Hayat, H. Yasmin and M. Al-Yami, Soret and Dufour effects in peristaltic transport of physiological fluids with chemical reaction: A mathematical analysis, Computers \& Fluids 89 (2014), 242-253.

[23] N.S. Akbar, S. Nadeem and C. Lee, Peristaltic flow of a prandtl fluid in an asymmetric channel, International Journal of Physical Sciences 7 (2012), 687-695.

[24] G. Sucharitha, S. Sreenadh and P. Lakshminarayana, Nonlinear peristaltic transport of a conducting prandtl fluid in a porous asymmetric channel, International Journal of Engineering Research and Technology 1 (2012) ISSN: 2278-0181.

[25] S. Navaneeswara Reddya, G. Viswanatha Reddyb and M.V. Subba Reddy, Peristaltic flow of a prandtl fluid through a porous medium in a channel, International Journal of Mathematical Archive 11 (2012), 4072-4080.

[26] S. Jothia, A.R. Prasad and M.V. Subba Reddy, Peristaltic flow of a prandtl fluid in a symmetric channel under the effect of a magnetic field, Advances in Applied Science Research 3 (2012), 2108-2119.

[27] L.M. Srivastava and V.P. Srivastava, Peristaltic transport of a power law fluid: Applications to the ductus efferentes of the reproductive tract, Rheologica Acta 27 (1988), 428-433.

[28] H.S. Lew, Y.C. Fung and C.B. Lowenstein, Peristaltic carrying and mixing of chyme, Journal of Biomechanics 4 (1971), 297-315.

[29] O. Eytan and D. Elad, Analysis of intra-uterine fluid motion induced by uterine contractions, Bulletin of Mathematical Biology 61 (1999), 221-238.

[30] H. Strohmer, A. Obruca, K.M. Rander and W. Feichtinger, Relationship of the individual uterine size and the endometrial thickness in stimulated cycles, Fertility and Sterlity 61 (1994), 972-975.

\section{Appendix A}

We describe here the quantities appeared in the solutions.

$$
\begin{aligned}
A_{0}= & \left(\left(E_{5}-4 \pi^{2}\left(E_{1}+E_{2}-4 E_{4} \pi^{2}\right)\right)\right. \\
& \left.\cos [2 \pi(x-t)]+2 E_{3} \pi \sin [2 \pi(x-t)]\right), \\
A_{1}= & -2 A_{0} \pi \varepsilon / M^{2}, \\
A_{2}= & \left(2 A_{0} \sqrt{\alpha} \pi \varepsilon \sec h[M+M \varepsilon \sin [2 \pi(x-t)]]\right) / M^{3}, \\
B_{0}= & \sec h(M \eta / \sqrt{\alpha})^{4}, \\
B_{1}= & \cosh (2 M(-1+\varepsilon \sin [2 \pi(t-x)]) / \sqrt{\alpha}), \\
B_{2}= & \cosh (4 M(-1+\varepsilon \sin [2 \pi(t-x)]) / \sqrt{\alpha}), \\
B_{3}= & \cosh (M \eta / \sqrt{\alpha}),
\end{aligned}
$$


$B_{4}=\cosh (3 M(-1+\varepsilon \sin [2 \pi(t-x)]) / \sqrt{\alpha}), \quad+\alpha \cosh (M \eta / \sqrt{\alpha})\left(B i_{2}+B i_{1}\left(1+2 \eta B i_{2}\right)\right)$

$B_{5}=(-1+\varepsilon \sin [2 \pi(t-x)]) \sinh (M \eta / \sqrt{\alpha})$,

$$
L_{1}=\frac{A_{0}^{2} B r \pi^{2} \epsilon^{2} \sqrt{B_{0}}(2 M \eta-\sqrt{\alpha} \sinh (M \eta / \sqrt{\alpha}))\left(B i_{1}-B i_{2}\right)}{M^{3}\left(B i_{2}+B i_{1}\left(1+2 \eta B i_{2}\right)\right)}
$$

$L_{2}=\frac{1}{M^{2}} \times A_{0}^{2} B r \pi^{2} \epsilon^{2} \sec h(M \eta / \sqrt{\alpha})^{2}$,

$L_{3}=\frac{-1}{2 M^{4}} \times A_{0}^{2} B r \pi^{2} \alpha \epsilon^{2} \sec h(M \eta / \sqrt{\alpha})^{2}$,

$L_{4}=\frac{A_{0}^{2} B r \pi^{2} \epsilon^{2} \sec h[M \eta / \sqrt{\alpha}]^{2}}{2 M^{4}\left(B i_{2}+B i_{1}\left(1+2 \eta B i_{2}\right)\right)}$

$\left(2 M \sqrt{\alpha} \sinh (M \eta / \sqrt{\alpha})\left(2+\eta\left(B i_{1}+B i_{2}\right)\right)\right.$
Similarly the constants $D_{k}(k=1-13)$ in Equations (45-47) can be obtained by using boundary conditions (32-35) and (41-44) through algebraic computations. 



Submit your manuscripts at

http://www.hindawi.com
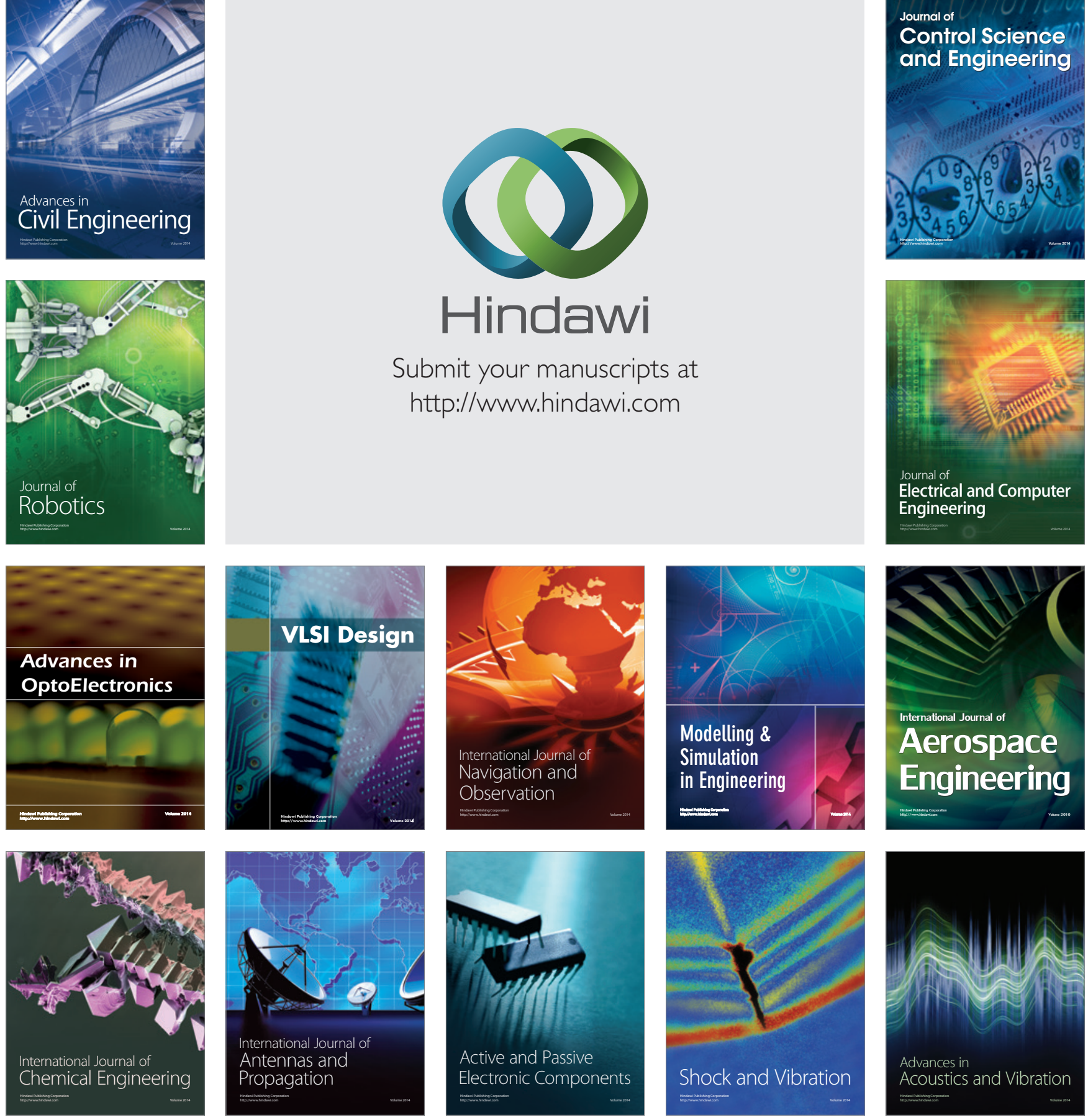